KEK-CP-351, OU-HET-921

\title{
Approaching the Bottom Using Fine Lattices With Domain-Wall Fermions
}

\author{
Brendan Fahy $^{* a}$, Guido Cossu ${ }^{a}$, Shoji Hashimoto ${ }^{a b}$ \\ ${ }^{a}$ High Energy Accelerator Research Organization (KEK), Ibaraki 305-0801, Japan \\ ${ }^{b}$ School of High Energy Accelerator Science, SOKENDAI (The Graduate University for \\ Advanced Studies), Tsukuba 305-0801, Japan
}

\begin{abstract}
We explore the heavy-quark mass region above the charm mass using Möbius domain-wall fermions on fine lattices at $a=0.080,0.055$, and $0.044 \mathrm{fm}$. We examine masses and decay constants using a series of heavy quark masses up to 3 times the charm quark. We analyze the cutoff effects for heavy quarks above the charm and account for the leading order discretization effects using ideas from HQET. We extrapolate to the bottom quark mass and report preliminary results for $f_{B}$ and $f_{B_{s}}$.
\end{abstract}

34th annual International Symposium on Lattice Field Theory

24-30 July 2016

University of Southampton, UK

\footnotetext{
*Speaker.
} 


\section{Introduction and Lattice Setup}

Direct lattice simulation of the bottom quark is still a challenge for lattice QCD. One approach is to use effective actions specific for heavy quark physics such as non-relativistic QCD and match back to QCD. Modern lattices are, on the other hand, much finer than in the past and cutoff effects at the charm are small. Using very fine lattices, cutoff effects are manageable even above the charm mass [1], which allows us to produce results between the charm and the bottom with enough points to extrapolate to the bottom.

The JLQCD collaboration has recently produced lattice ensembles with $2+1$ flavors of Möbius domain-wall fermions [2]. The gauge action used is tree-level improved Symanzik. These lattices have relatively fine lattice spacings of $1 / a \approx 2.4,3.6$, and $4.5 \mathrm{GeV}$ with pion masses between 230 $\mathrm{MeV}$ and $500 \mathrm{MeV}$ [3]. The lattice spacing was determined from Wilson flow using $t_{0}$ with the physical value from [4]. The parameters of each of the 15 lattices can be found in Table 1.

Axial and pseudo-scalar two-point correlators were computed with the Iroiro++ software package [5]. Correlators were improved using $Z_{2}( \pm 1)$ noise sources distributed over a single time slice, and sources computed on many time slices on a single configuration are then averaged. A total of $400-600$ measurements were carried out as detailed in Table 1. Each of these $Z_{2}$ sources were computed both unsmeared and with Gaussian smearing. The source-sink combinations of unsmeared-smeared and smeared-smeared were simultaneously fit to extract meson masses and decay constants. Due to good chiral symmetry of our domain-wall fermions the decay constants can be computed directly from the psuedo-scalar currents utilizing the PCAC relation. These lattices have also been used to compute semi-leptonic $D$ decays in [6] and charm quark mass determination from short-distance correlators in [7].

\section{Charm Results}

The first goal was to determine the decay constants $f_{D}$ and $f_{D_{s}}$. These were determined from pseudo-scalar correlators at the charm mass determined from a previous study [1] with an input of the spin averaged $c \bar{c}$ mass. The values for $f_{D_{(s)}}$ for all of our ensembles can be seen in Figure 1. We preformed a global fit to all the ensembles assuming linear dependence on the light and strange quark masses and on the lattice spacing squared. The plots show lines corresponding to the continuum limit (black) as well as the line evaluated at a lattice spacing corresponding to our coarsest lattices. The difference between the continuum limit and our coarsest lattice is roughly $2 \%$. The plots for $f_{D_{s}}$ (right panel) have fit lines which do not go through the cluster of points because the data are simulated at strange quark masses which sandwich the physical value.

The results of the global fit evaluated at the physical point are

$$
f_{D}=212.8 \pm 1.7 \pm 3.6 \mathrm{MeV}, \quad f_{D_{s}}=244.0 \pm 0.84 \pm 4.1 \mathrm{MeV},
$$

with the errors being the statistical error and the systematic error from the scale determination, respectively. 


\begin{tabular}{|l|l|r|r|r|r|r|r|}
\hline$\beta$ & $L^{3} \times T$ & $L_{5}$ & $a m_{\text {ud }}$ & $a m_{s}$ & $\begin{array}{r}m_{\pi} \\
{[\mathrm{MeV}]}\end{array}$ & $m_{\pi} L$ & \#meas \\
\hline$\beta=4.17$ & $32^{3} \times 64$ & 12 & 0.0035 & 0.040 & 230 & 3.0 & 800 \\
& & & 0.0070 & 0.030 & 310 & 4.0 & 800 \\
& & & 0.0070 & 0.040 & 310 & 4.0 & 800 \\
& & & 0.0120 & 0.030 & 400 & 5.2 & 800 \\
& & & 0.0120 & 0.040 & 400 & 5.2 & 800 \\
& & & 0.0190 & 0.030 & 500 & 6.5 & 800 \\
& & & 0.0190 & 0.040 & 500 & 6.5 & 800 \\
\cline { 2 - 7 }$\beta=4.35$ & $48^{3} \times 96$ & 12 & 0.0035 & 0.040 & 230 & 4.4 & 800 \\
\hline & $48^{3} \times 96$ & 8 & 0.0042 & 0.018 & 300 & 3.9 & 600 \\
& & & 0.0042 & 0.025 & 300 & 3.9 & 600 \\
& & & 0.0080 & 0.018 & 410 & 5.4 & 600 \\
& & & 0.0080 & 0.025 & 410 & 5.4 & 600 \\
& & & 0.0120 & 0.018 & 500 & 6.6 & 600 \\
& & & 0.0120 & 0.025 & 500 & 6.6 & 600 \\
\hline$\beta=4.47$ & $64^{3} \times 128$ & 8 & 0.0030 & 0.015 & 280 & 4.0 & 400 \\
\hline
\end{tabular}

Table 1: Parameters of the JLQCD gauge ensembles used in this work. Pion masses are rounded to the nearest $10 \mathrm{MeV}$. Inverse lattice spacings are $a^{-1}=2.453(4) \mathrm{GeV}, 3.610(9) \mathrm{GeV}$, and 4.496(9) GeV for $\beta=4.17,4.35$ and 4.47 respectively. The $L_{5}$ length in the domain-wall is 12 at $\beta=4.17$ and 8 on the finer lattices. The spatial extent satisfies $m_{\pi} L \gtrsim 4.0$ for all lattices except the first lattice in the table, which was excluded from the final analysis in this work. The number of measurements listed in the final column are the product of the number of configurations used and the number of time sources on each configurations. 100 configuration's were used for the $\beta=4.17$ ensembles and 50 in the others.

\section{Above Charm}

Since the cutoff effects at the charm mass were small we expect that the results at slightly heavier quark masses are also under control. We produced heavy-light and heavy-strange correlators using a set of heavy masses above the charm mass. These were chosen to be above the charm mass by factors of 1.25 producing a sequence $m_{n}=(1.25)^{n} m_{c}$. The bare charm mass is determined in a separate study [1]. The values for $m_{n}$ used on different $\beta$ values are shown in Table 2. For the finer lattices we produced five bare quark masses above the charm mass, while we limited to only three masses above charm for our coarsest lattice as the bare quark mass $(1.25)^{4} m_{c}$ exceeds 1.0. Note that the maximum possible mass for the domain wall fermion is given by the Pauli-Villars mass, which is 1 in the standard implementation.

To see how well things scaled from the charm towards the bottom we looked at the matrix element $f_{h x} \sqrt{m_{h x}}$ where $f$ is the pseudo-scalar decay constant, $m$ is the pseudo-scalar meson mass, and $x$ is either a strange $(s)$ quark or light $(\ell)$ quark. This was chosen because in the heavy quark limit, $m_{h} \rightarrow \infty$, the combination $f_{h x} \sqrt{m_{h x}}$ is known to scale as a constant up to the anomalous dimension contribution (see below). Plots of this verses the inverse meson mass, for heavy-light (left) or heavy-strange (right), are shown in Figure 2. The results on all ensembles are plotted together with the cluster of points on the right side being the values at the charm mass and moving 

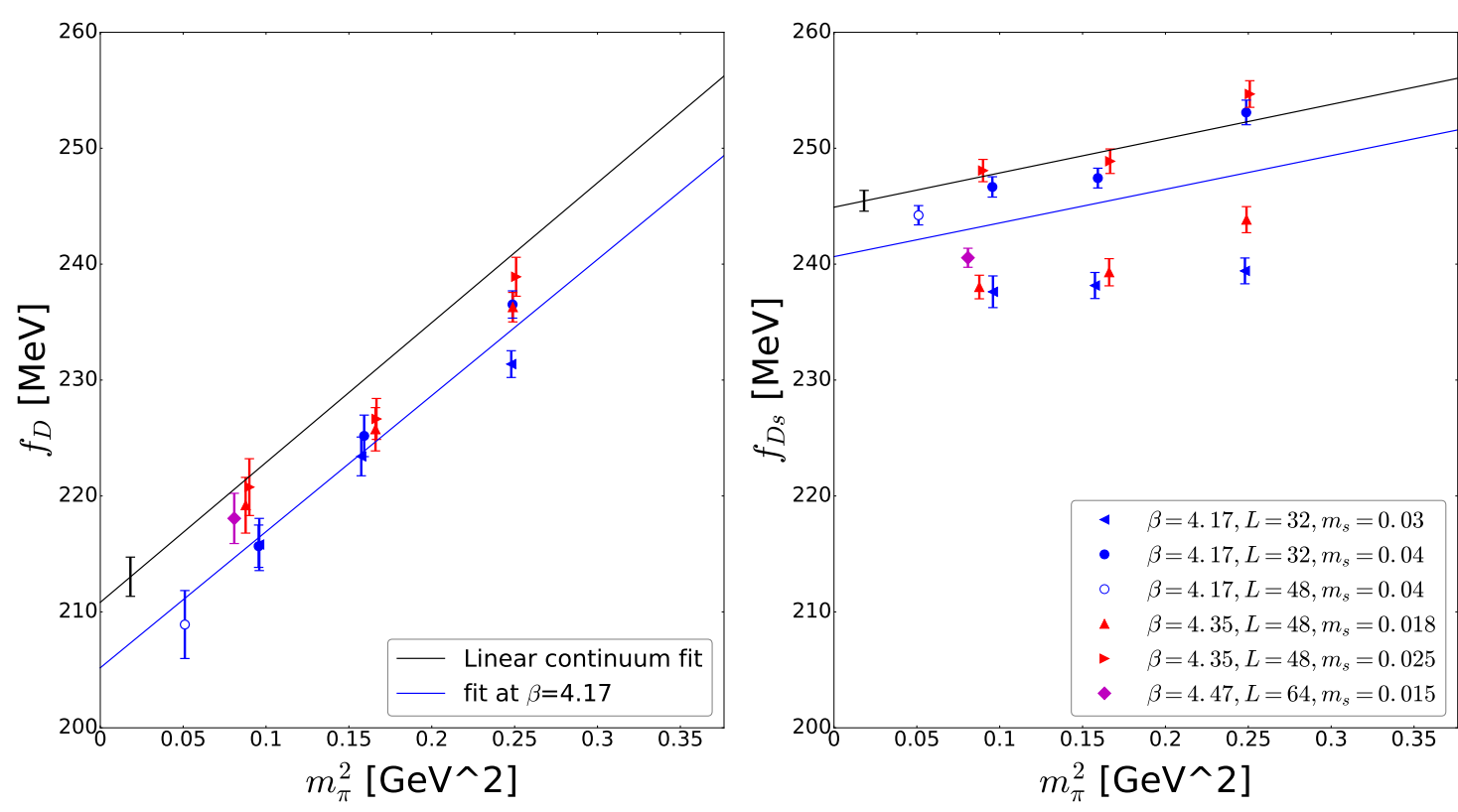

Figure 1: Plots of $f_{D}$ (left) and $f_{D_{s}}$ (right) vs $m_{\pi}^{2}$. The fit lines correspond to the continuum limit (black) and the coarsest lattice (blue). They are linear in $m_{\pi}^{2}$ and are interpolated to the physical strange point using $2 m_{K}^{2}-m_{\pi}^{2}$.

\begin{tabular}{|l|r|r|r|r|r|r|}
\hline Beta & $m_{0}=m_{c}$ & $m_{1}$ & $m_{2}$ & $m_{3}$ & $m_{4}$ & $m_{5}$ \\
\hline 4.17 & 0.4404 & 0.5505 & 0.6881 & 0.8600 & & \\
4.35 & 0.2729 & 0.3411 & 0.4264 & 0.5330 & 0.6661 & 0.8327 \\
4.45 & 0.2105 & 0.2631 & 0.3289 & 0.4111 & 0.5139 & 0.6423 \\
\hline
\end{tabular}

Table 2: Bare heavy quark masses where $m_{0}$ is the charm quark mass $m_{n}=\lambda^{n} m_{0}$.

to the left each cluster being the next choice for the heavy quark mass. It is clear that on the coarsest lattice (blue) discretization effects become significant already at $(1.25)^{2} m_{c}$ and heavier. In the next section we attempt to account for the leading order $a^{2}$ dependence. Here as a first attempt we simply perform a global fit to all of the data including a term to account for $a^{2}$ as well as $a^{2} m^{2}$ effects.

The global fit is performed using an ansatz

$$
\begin{aligned}
& f_{h x} \sqrt{m_{h x}}=\left(\Phi_{\text {phys }}\right)\left(1+\frac{C_{1}}{m_{h}}+\frac{C_{2}}{m_{h}^{2}}\right) \\
& \Phi_{\text {phys }}=\left(1+\gamma_{S}\left(2 M_{K}^{2}-M_{\pi}^{2}\right)+\gamma_{P}\left(M_{\pi}^{2}-\left(M_{\pi}^{\text {phys }}\right)^{2}\right)+\gamma_{A} a^{2}+\gamma_{M A}(m a)^{2}\right) .
\end{aligned}
$$

The basic assumption is the dependence on the inverse meson mass is a polynomial preserving a constant in the limit of $m \rightarrow \infty$. The $\Phi_{\text {phys }}$ accounts for the extrapolation to the physical pion mass, interpolation to the physical strange quark mass using $2 M_{K}^{2}-M_{\pi}^{2}$, and extrapolation to the cotinuum limit with both $m^{2} a^{2}$ and $a^{2}$ terms. Here the fit excludes the heaviest points on the $\beta=4.17$ and $\beta=4.35$ lattices which have a bare quark mass above 0.8 where the discretization effect of order $(\mathrm{ma})^{4}$ and higher are expected to be more significant. The fit curves corresponding to each $\beta$ value 

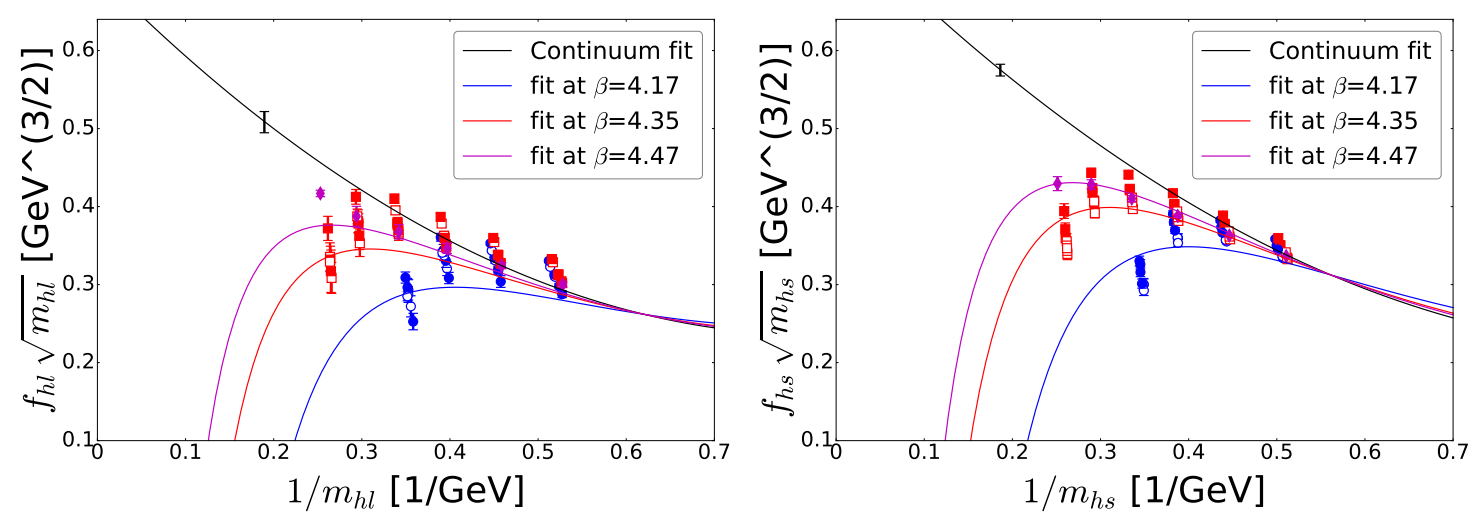

Figure 2: Values for $f \sqrt{m}$ for heavy-light (left) and heavy-strange (right) on all of our ensembles for each of the different heavy-quark masses from Table 2. Data points are those of $\beta=4.17$ (blue), 4.35 (red) and 4.47 (magenta). Each horizontal cluster of points shows values for a particular choice of heavy quark mass with the value at the charm mass on the far right and increasing heavy quark masses going to the left. The black line indicates a global fit in physical limit as discussed in the text. The colored lines indicate the same fit parameters evaluated at the finite lattice spacings corresponding to our three choices of $\beta$. The empty points with error bars show the statistical uncertainly of the fit at the physical values for $m_{B}$ and $m_{B_{s}}$.

and physical light/strange masses are shown in Figure 2, We observe that the data points drift away from the continuum curve (black) as ma gets large. In particular the heaviest points at $\beta=4.17$ and 4.35 suffer from strong discretization effects.

\section{HQET Corrections}

To understand the leading order cutoff effects we use ideas from Heavy Quark Effective Theory (HQET). We closely follow the discussion of $[8,9]$, which was mainly applied to the Wilsontype fermions.

Expanding the energy of a free quark for low momentum we obtain $E \approx m_{1}+\frac{p^{2}}{2 m_{2}}+\ldots$, where on the lattice the "rest mass", $m_{1}$, may not be equal to the "kinetic mass", $m_{2}$. These corrections were computed years ago for Wilson fermions [8] which give simple corrections that have been used to design actions suited for heavy quarks [9]. In the case of domain wall fermions the expressions for these corrections are not as simple.

Starting with the propagator for domain-wall fermions [10] and expanding in low momentum we obtain $m_{1}$ and $m_{2}$ at tree level as well as the wave-function renormalization, $A_{K L M}^{D W}$. The expressions for these factors are,

$$
\begin{array}{cc}
m_{1} & =\log \left(1-W_{0}+\sqrt{\left(1-W_{0}\right)^{2}-1}\right), \\
m_{2}= & \sqrt{W_{0}^{2}-2 W_{0}}\left(\frac{Q+1-2 W_{0}}{(Q+1)+(Q-1)\left(2 W_{0}^{2}+W_{0}\right)}\right), \\
A_{K L M}^{D W} & =\frac{2}{\left(1-m^{2}\right)\left[1+\sqrt{\frac{Q}{1+4 W_{0}}}\right]},
\end{array}
$$



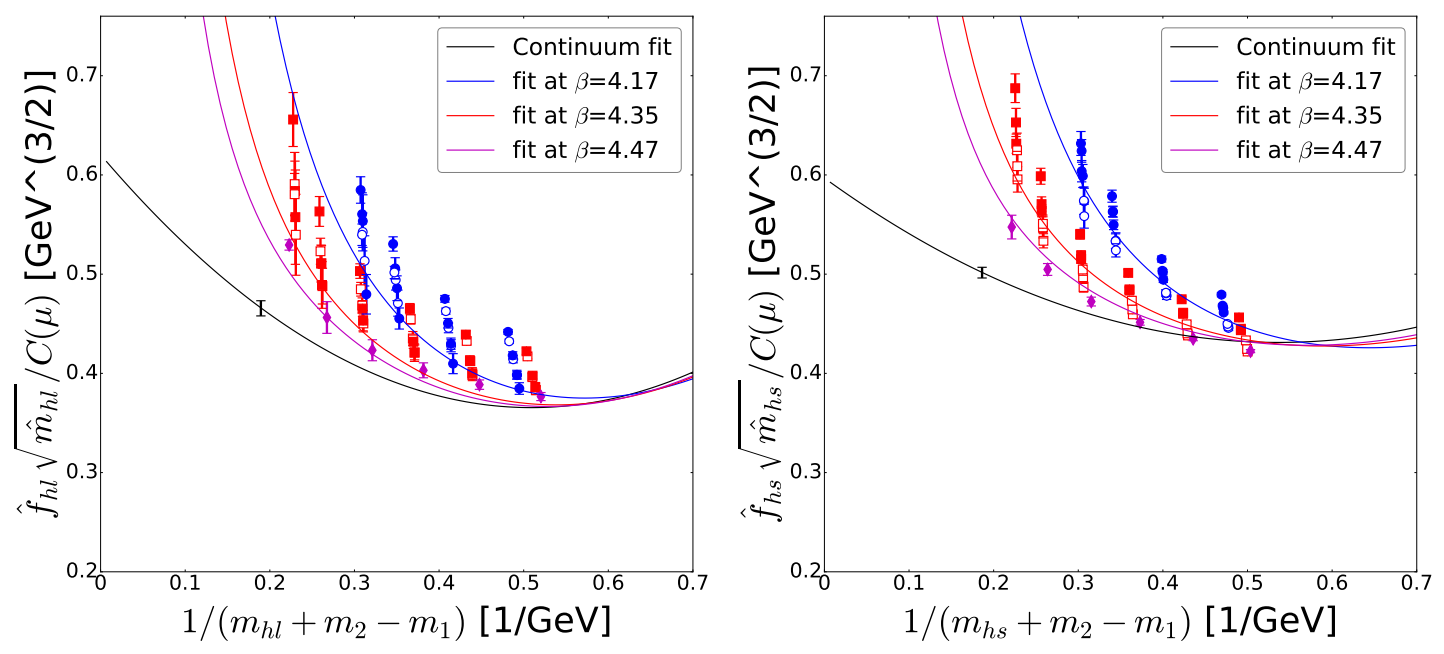

Figure 3: Same as Figure 2 with the corrections described in Section 4. The anomalous dimension factors are also included. The fit now accounts for leading order $a^{2} m^{2}$ effects so the correction used is $\gamma_{M A} \alpha_{s}(m a)^{2}$. The black error bars on the fit give the statistical uncertainty at the $B$ and $B_{s}$ meson mass respectively.

where

$$
Q=\left(\frac{1+m^{2}}{1-m^{2}}\right)^{2} \quad \text { and } \quad W_{0}=\frac{1+Q}{2}-\frac{\sqrt{3 Q+Q^{2}}}{2}
$$

According to $[8,9]$ we can then re-scale the heavy quark mass in favor of $m_{2}$ from $m_{1}$ by adding $m_{2}-m_{1}$, since the kinetic mass controls the motion of the heavy quark inside the meson. Similarly, we divide the amplitude by $A_{K L M}^{D W}$ to eliminate the leading discretization effect for the heavy quark.

These corrections, however, turned out to be insufficient to account for the heavy quarks propagator at short distances. If we numerically integrate the free propagator and divided by $A_{K L M}$, they agree at large time separations but for small separation they disagree. This deviation from a simple exponential behavior may be due to the non-locality of domain-wall fermions, which becomes relatively more significant at large quark masses. To fully capture such lattice artifacts we numerically integrate the free propagator for each bare heavy quark mass, and divide our correlators by these and multiply back by the continuum result, so that the heavy quark propagator coincides that of the continuum at least at tree level.

Applying these corrections we match the decay constants from QCD to HQET with a factor accounting for the anomalous dimension $C(\mu)$. Perturbative calculation of $C(\mu)$ is available up to three loop, $\alpha_{s}^{3}$ [11]. These results and fit are shown in Figure 3. These results exhibit less divergence from the continuum limit of the values for the heaviest quark masses indicating that we have successfully account for the bulk of the leading $a^{2} m^{2}$ cutoff effects. The global fit to the corrected data is performed in the same manner as before, see (3.1), but replacing the $m^{2} a^{2}$ term with $\gamma_{M A} \alpha_{s}(m a)^{2}$ as we have accounted for the tree level corrections. The continuum limit (black line) as well as the fit for the individual finite lattice spacings (colored lines) are plotted. The fit function evaluated at the $B$ and $B_{s}$ mass yields,

$$
f_{B}=195.5 \pm 3.2 \pm 3.3 \mathrm{MeV}, \quad \text { and } \quad f_{B_{s}}=218.2 \pm 1.9 \pm 3.7 \mathrm{MeV} .
$$


These results are within $2 \sigma$ of the current FLAG average [12].

\section{Summary}

Using fine lattices we are able to obtain results for pseudo-scalar decay constants above the charm mass at nearly the bottom mass. Accounting for the leading discretization effects we are able to extrapolate to the bottom quark mass and predict $f_{B}$ and $f_{B_{s}}$. This is all done without requiring a specialized action for heavy quarks as they were treated with the same domain wall action used for light quarks.

Numerical simulations are performed on Hitachi SR16000 and IBM System Blue Gene Solution at KEK under the support of its Large Scale Simulation Program (No. 16/17-14). This research is supported in part by the Grant-in-Aid of the MEXT (No. 26247043, 25800147) and by MEXT as "Priority Issue on Post-K computer" (Elucidation of the Fundamental Laws and Evolution of the Universe) and JICFuS.

\section{References}

[1] JLQCD collaboration, B. Fahy, G. Cossu, S. Hashimoto, T. Kaneko, J. Noaki and M. Tomii, Decay constants and spectroscopy of mesons in lattice QCD using domain-wall fermions, POS (LATTICE $2015) 074$ (2016).

[2] R. C. Brower, H. Neff and K. Orginos, The Möbius Domain Wall Fermion Algorithm, 1206.5214.

[3] J. Noaki, S. Aoki, G. Cossu, S. Fukaya, H. Hashimoto and T. Kaneko, Fine lattice simulations with chirally symmetric fermions, POS (LATTICE 2013) 263 (2014).

[4] S. Borsányi, S. Dürr, Z. Fodor, C. Hoelbling, S. Katz, S. Krieg et al., High-precision scale setting in lattice QCD, Journal of High Energy Physics 2012 (2012).

[5] G. Cossu, J. Noaki, S. Hashimoto, T. Kaneko, H. Fukaya, P. Boyle et al., JLQCD IroIro++ lattice code on BG/Q, POS (LATTICE 2013) 482 (2014).

[6] JLQCD collaboration, T. Kaneko, B. Fahy, H. Fukaya and S. Hashimoto, D meson semileptonic decays in lattice QCD with Moebius domain-wall quarks, POS (LATTICE 2016) 297 (2016).

[7] JLQCD collaboration, K. Nakayama, B. Fahy and S. Hashimoto, Short-distance charmonium correlator on the lattice with möbius domain-wall fermion and a determination of charm quark mass, Phys. Rev. D 94 (Sep, 2016) 054507.

[8] G. Lepage, Simulating heavy quarks, Nuclear Physics B - Proceedings Supplements 26 (1992) 45 56.

[9] A. X. El-Khadra, A. S. Kronfeld and P. B. Mackenzie, Massive fermions in lattice gauge theory, Phys. Rev. D 55 (Apr, 1997) 3933-3957.

[10] S. Aoki, T. Izubuchi, Y. Kuramashi and Y. Taniguchi, Perturbative renormalization factors of quark bilinear operators for domain-wall qcd, Phys. Rev. D 59 (1999) 094505.

[11] S. Bekavac, A. Grozin, P. Marquard, J. Piclum, D. Seidel and M. Steinhauser, Matching QCD and HQET heavy-light currents at three loops, Nuclear Physics B 833 (2010) 46 - 63.

[12] S. Aoki, Y. Aoki, C. Bernard, T. Blum, G. Colangelo, M. Della Morte et al., Review of lattice results concerning low-energy particle physics, The European Physical Journal C 74 (2014). 